Article

\title{
Epitaxial Silver Films Morphology and Optical Properties Evolution over Two Years
}

\author{
Aleksandr S. Baburin $1,2,+$, Anton I. Ivanov $1,2,+$, Evgeniy S. Lotkov ${ }^{1,2} \mathbb{D}$, Olga S. Sorokina ${ }^{1,2}$, \\ Irina A. Boginskaya ${ }^{3}$, Evgeniy V. Sergeev ${ }^{1,2}$, Kirill A. Buzaverov ${ }^{1,2}$, Tatiana G. Konstantinova ${ }^{1}$, \\ Dmitriy O. Moskalev ${ }^{1,2}$, Zhamila Issabayeva ${ }^{1}$, Ilya A. Ryzhikov ${ }^{1,3}$ and Ilya A. Rodionov $1,2, *$ (D) \\ 1 FMN Laboratory, Bauman Moscow State Technical University, 105005 Moscow, Russia; \\ baburin@bmstu.ru (A.S.B.); aivanov@bmstu.ru (A.I.I.); lotevg@bmstu.ru (E.S.L.); Soros@bmstu.ru (O.S.S.); \\ sergeev_e@bmstu.ru (E.V.S.); kirillbuz@gmail.com (K.A.B.); tgkonst@bmstu.ru (T.G.K.); \\ Moskalevdm@bmstu.ru (D.O.M.); zhamilyaiss@gmail.com (Z.I.); ilyaryzhikov@rambler.ru (I.A.R.) \\ 2 Dukhov Automatics Research Institute, (VNIIA), 127055 Moscow, Russia \\ 3 Institute for Theoretical and Applied Electromagnetics RAS, 125412 Moscow, Russia; i.boginskaya@bk.ru \\ * Correspondence: irodionov@bmstu.ru \\ + These authors contributed equally to this work.
}

Received: 27 August 2020; Accepted: 21 September 2020; Published: 23 September 2020

check for updates

\begin{abstract}
Silver and gold are the most commonly used materials in optics and plasmonics. Silver has the lowest optical losses in the visible and near-infrared wavelength range, but it faces a serious problem-degradation over time. It has been repeatedly reported that the optical properties of silver thin films rapidly degrade when exposed to the atmosphere. This phenomenon was described by various mechanisms: rapid silver oxidation, sorption of sulfur or oxygen, formation of silver compounds with chlorine, sulfur, and oxygen. In this work, we systematically studied single-crystalline silver films from 25 to $70 \mathrm{~nm}$ thicknesses for almost two years. The surface morphology, crystalline structure and optical characteristics of the silver films were measured using spectroscopic ellipsometry, ultra-high-resolution scanning electron microscopy, and stylus profilometry under standard laboratory conditions. After 19 months, bulk structures appeared on the surface of thin films. These structures are associated with relaxation of internal stresses combined with dewetting. Single-crystalline silver films deposited using the single-crystalline continuous ultra-smooth, low-loss, low-cost (SCULL) technology with a thickness of 35-50 nm demonstrated the best stability in terms of degradation. We have shown that the number of defects (grain boundaries and joints of terraces) is one of the key factors that influence the degradation intensity of silver films.
\end{abstract}

Keywords: single-crystalline thin film; over-time research; structural degradation; ellipsometry; scanning electron microscopy

\section{Introduction}

Silver, aluminum, and gold thin films are the key elements of devices in various modern fields of research [1-3]. The range of their applications covers both integral plasmonic devices and computing elements [4-7] as well as various functional coatings (e.g., for optical mirrors [8]). Among the mentioned materials, silver has the lowest losses in the visible and near-infrared ranges $[2,9,10]$. Silver films are crucial for many novel devices like spasers [11,12], sensors [13-15] nano-antennas [16,17], metasurfaces [18], etc. However, silver films suffer from the well-known problem of optical degradation over time. As a result, they are only used in short lifetime devices or laboratory experiments. Nowadays, the most popular material for the devices mentioned above is gold due to its excellent stability under environmental conditions [19]. 
Studying the silver degradation mechanism and looking for a proper solution to prevent it is an important problem, especially for epitaxial films, where an atomically smooth surface and perfect crystalline structure play a key role [20]. Over the past decades, many experiments were carried out to investigate the degradation mechanisms. However, there is still no common theory and accurate description of this phenomenon. It is considered that the main reasons for degradation of thin silver films are the sorption of oxygen, sulfur, and chlorine from the atmosphere followed by the formation of chemical compounds with silver [21-25]. However, these chemical compounds were not found in many studies of films and silver particles [26,27]. Some works stress the role of the $\mathrm{H}_{2} \mathrm{O}$ layer in the chemical reaction of the degradation process leading to the dissociation of silver atoms [28]. Dewetting of silver layers was also considered in some published papers [27,29-31]. These studies demonstrate that silver degradation can be promoted by surface tension forces induced in silver thin films during deposition. One of the common approaches to protect silver from degradation is using top and under layers [27,32], but it could be critical in terms of optical performance.

In this work, we have studied single-crystalline silver films of different thicknesses over two years. We demonstrated the long-time evolution of the silver surface morphology and optical characteristics under standard laboratory conditions, proving that high-quality crystalline structure and perfect surfaces can significantly reduce degradation rate.

\section{Materials and Methods}

\subsection{Preparation of Thin Films}

Four silver films of different thicknesses $(25,35,50,70 \mathrm{~nm})$ were deposited on $<111>$ silicon substrates by a $10 \mathrm{~kW}$ e-beam evaporator (Angstrom Engineering) with the base pressure of less than $3 \times 10^{-8}$ Torr. First, the substrates were cleaned in a 2:1 sulfuric acid: hydrogen peroxide solution $\left(80^{\circ} \mathrm{C}\right)$, followed by cleaning in isopropanol to remove organics and native oxide in hydrofluoric acid. The purity of the silver used for evaporation was $99.999 \%$. The deposition technology of single-crystalline silver films was previously developed in our laboratory [33]. This deposition technology provides atomically smooth single-crystalline silver films deposition using two-step process. In the first step, a seed crystal consisting of strained two-dimensional islands with an atomically flat top surface is grown on a substrate under elevated temperature. As the result, the 2D islands seed is formed consisting of the uniform thickness islands with the atomically flat top surface. In the second step, the deposition is stopped and the substrate is cooled down to room temperature in the same vacuum cycle to prevent a well-known dewetting effect and three-dimensional growth leading to subsequent film imperfections. Then, more silver is deposited on the seed layer at room temperature until a continuous film is formed. Following film annealing under elevated temperature (higher than first step) can reduce growth defect density, improving the crystalline structure and surface roughness. With this dual-phase experimental nature in mind as well as improved film parameters, we name our deposition process the "SCULL" (Single-crystalline Continuous Ultra-smooth Low-loss Low-cost). Immediately after deposition, the samples structure was examined by scanning electron microscopy (SEM) (Figure 1). Also SEM images with higher magnifications are presented at Figure S1 (Supplementary Materials).

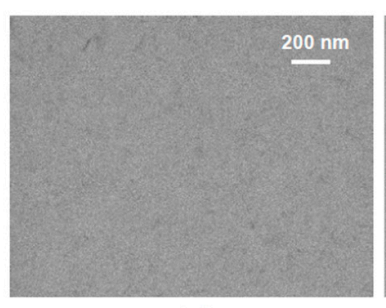

(a)

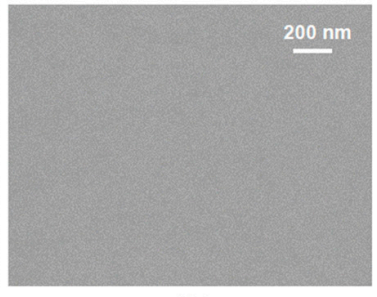

(b)

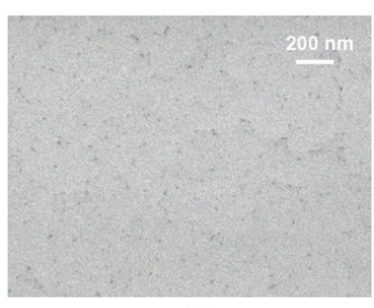

(c)

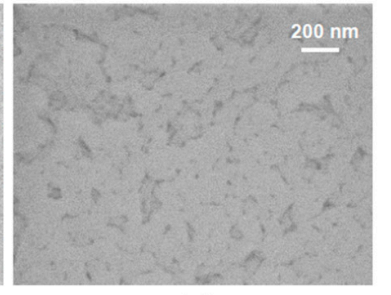

(d)

Figure 1. SEM surface images of: (a) $25 \mathrm{~nm}$ thickness film; (b) $35 \mathrm{~nm}$ thickness film; (c) $50 \mathrm{~nm}$ thickness film; (d) $70 \mathrm{~nm}$ thickness film. 


\subsection{Over-Time Characterization}

The samples were stored under controlled laboratory conditions (ISO6, humidity $45 \pm 5 \%$, temperature $\left.22 \pm 0.5^{\circ} \mathrm{C}\right)$ and characterized at regular intervals $(1 \mathrm{~h}, 3 \mathrm{~h}, 24 \mathrm{~h}, 1$ week, 2 weeks, 1 month, 2 months, 7.5 months, 19 months).

The dielectric functions of the silver films were measured by multi-angle spectroscopic ellipsometer (SER 800, Sentech GmbH, Berlin, Germany). Measured substrate optical constants and silver thickness were fixed in the subsequent data fitting for all the samples, and only silver parameters were allowed to change. Modeling and analysis were performed using SENresearch 4.0 software package (SER 800, Sentech $\mathrm{GmbH}$, Berlin, Germany). Measurement spectral wavelength range was from 330 to $1000 \mathrm{~nm}$, with an interval of approx. $2 \mathrm{~nm}$, and the reflected light was analyzed at incidence angles of $50^{\circ}, 60^{\circ}$, $70^{\circ}$. To characterize the optical losses, the real $\left(\varepsilon^{\prime}\right)$ and imaginary $\left(\varepsilon^{\prime \prime}\right)$ parts of the dielectric permittivity were extracted by fitting the measured raw ellipsometric data ( $\Psi$ and $\Delta$ ). In our fitting procedure we used the structural model of $\mathrm{Ag} / \mathrm{substrate}$ bilayer and simple phenomenological Brendel-Bormann (BB) oscillator model to interpret both the free electron and the interband parts of the dielectric response of our samples.

To check the quality and uniformity of the silver films, their surfaces were investigated using a Zeiss Merlin scanning electron microscope (Zeiss Microscopy GmbH, Jena, Germany). All the SEM images were obtained using in-lens detector and accelerating voltage of $5 \mathrm{kV}$, working distance from the sample to the detector was from 1 to $4 \mathrm{~mm}$. Magnifications of $3000 \times, 7000 \times, 15,000 \times$, and 50,000x were used to analyze the samples completely. To characterize the crystalline lattice orientation of the silver films electron backscatter diffraction (EBSD) maps were acquired for all the samples. The maps were obtained by Nordlys-II S electron back-scattering detector (EBSD, Oxford Instruments, Abingdon, $\mathrm{UK}$ ) at an accelerating voltage of $10 \mathrm{keV}$ at working distance of $12.5 \mathrm{~mm}$ and the sample tilt angle in the microscope chamber of $70^{\circ}$. To analyze the acquired EBSD maps AZtec 3.3 software (Oxford Instruments, Abingdon, UK) was used. The following initial values were chosen for the silver (Ag) phase: crystalline lattice-face-centered cubic (FCC); space group-225; diffraction symmetry class: $\mathrm{m} 3 \mathrm{~m}, \mathrm{a}, \mathrm{b}, \mathrm{c}=4.0855 \AA$, Alpha- $90^{\circ}$, Beta- $90^{\circ}$, Gamma- $90^{\circ}$, which gave the minimum deviation of the model from the measured data.

The surface roughness of the samples was characterized by means of KLA Tencor P17 stylus profiler (with a 38-nm Durasharp tip radius stylus, Milpitas, CA, USA). All measurements were performed using $0.5 \mathrm{mg}$ taping strength, the scan rate was $2 \mu \mathrm{m} / \mathrm{s}$, and the scanned line length was $20 \mu \mathrm{m}$.

\section{Results}

\subsection{Thin Film Surface Structure}

First, we investigated the films surface morphology evolution over time using a scanning electron microscope (Figure 2). One can find that after long-time storage (more than 7.5 months) cracks appear on almost all the silver films and thicker films have also volumetric inhomogeneities. These defects grow and become much bigger after 19 months in the atmosphere (SEM images of the $25 \mathrm{~nm}$ and $70 \mathrm{~nm}$ thick silver films are shown separately at lower magnifications (Figure 3)). We observed through-depth defects (holes) in $25 \mathrm{~nm}$ thick film (Figure 3a) and the emergence of voids (not full depth holes) in $70 \mathrm{~nm}$ thick film (Figure $3 \mathrm{~b}$ ). 


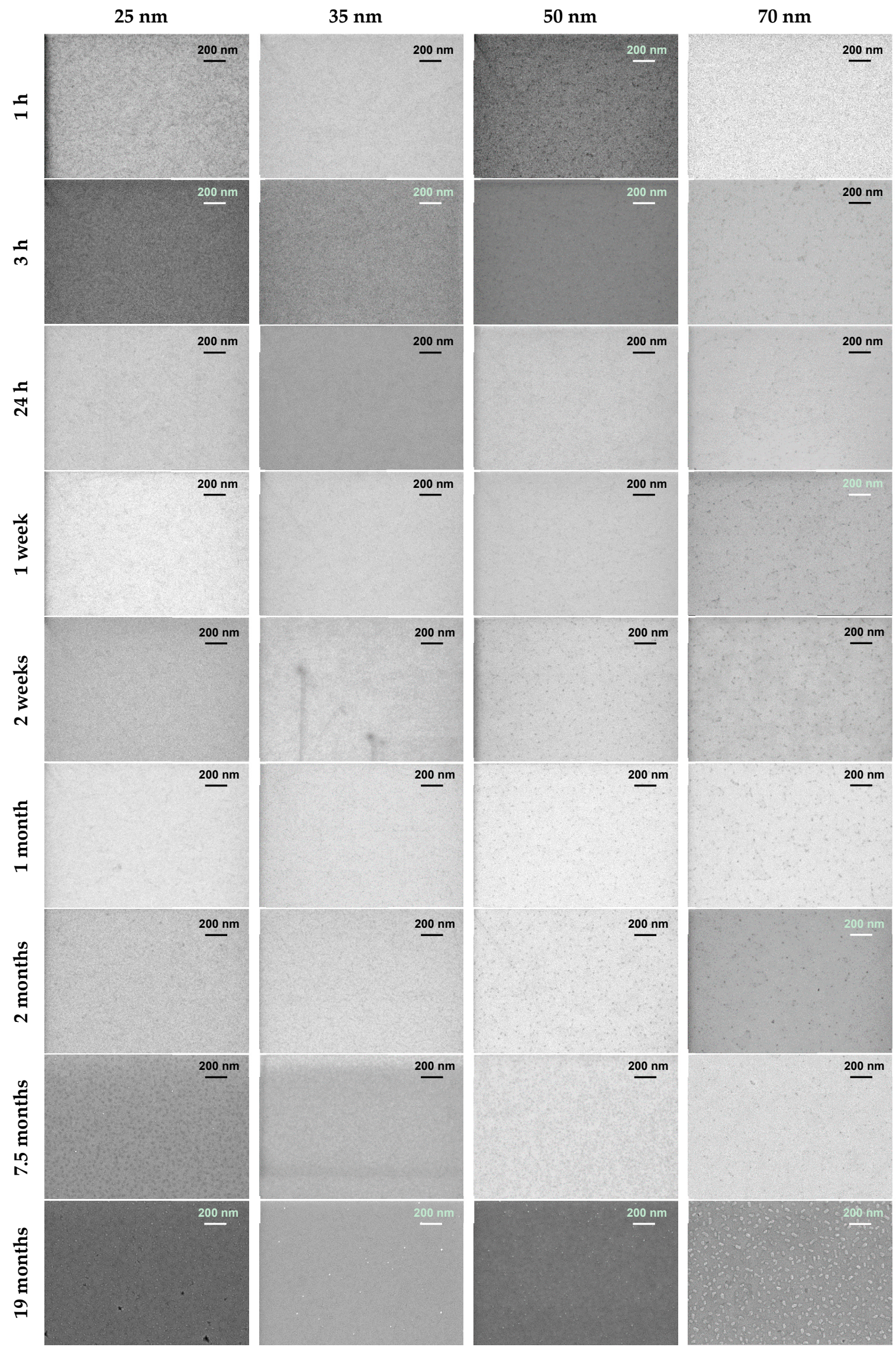

Figure 2. Over-time SEM surface images of the films (50,000× magnification). 


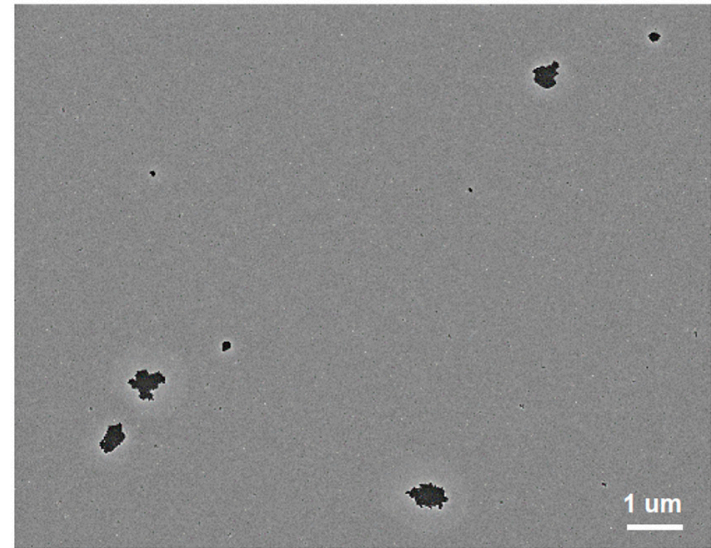

(a)

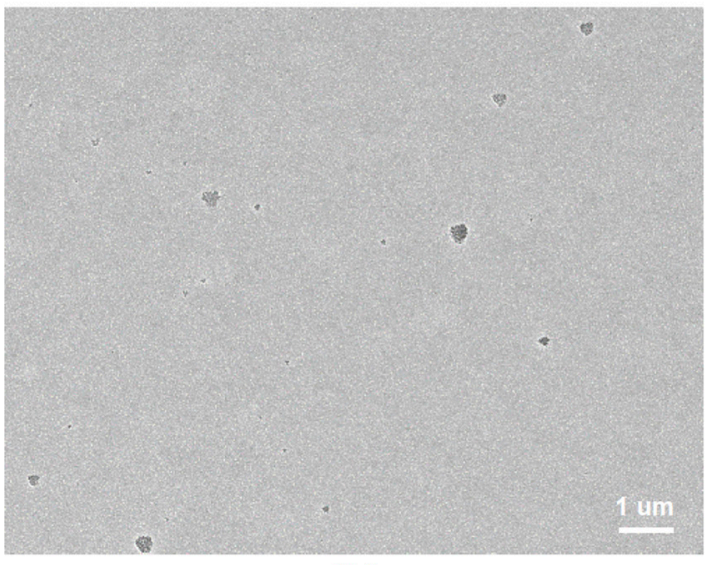

(b)

Figure 3. Over-time SEM surface images of Ag films after 19 months (7000× magnification): (a) $25 \mathrm{~nm}$, (b) $70 \mathrm{~nm}$.

In order to investigate the nature of these defects, and make some conclusions on the degradation mechanism (silver films material consumption due to chemical reactions, film surface roughness evolution) and assumptions on the layers stack for ellipsometry model, we measured all the samples using SEM at the cross section (Figure 4). No significant change in the average films thickness was found. However, after a certain period of time, above the silver film additional growing layers (additional roughness) was observed, which form an inhomogeneous structure in the form of protrusions. As it is shown below, these additional growing layers have nonmetallic optical properties pointing on the products of chemical reactions on the silver surface over time.
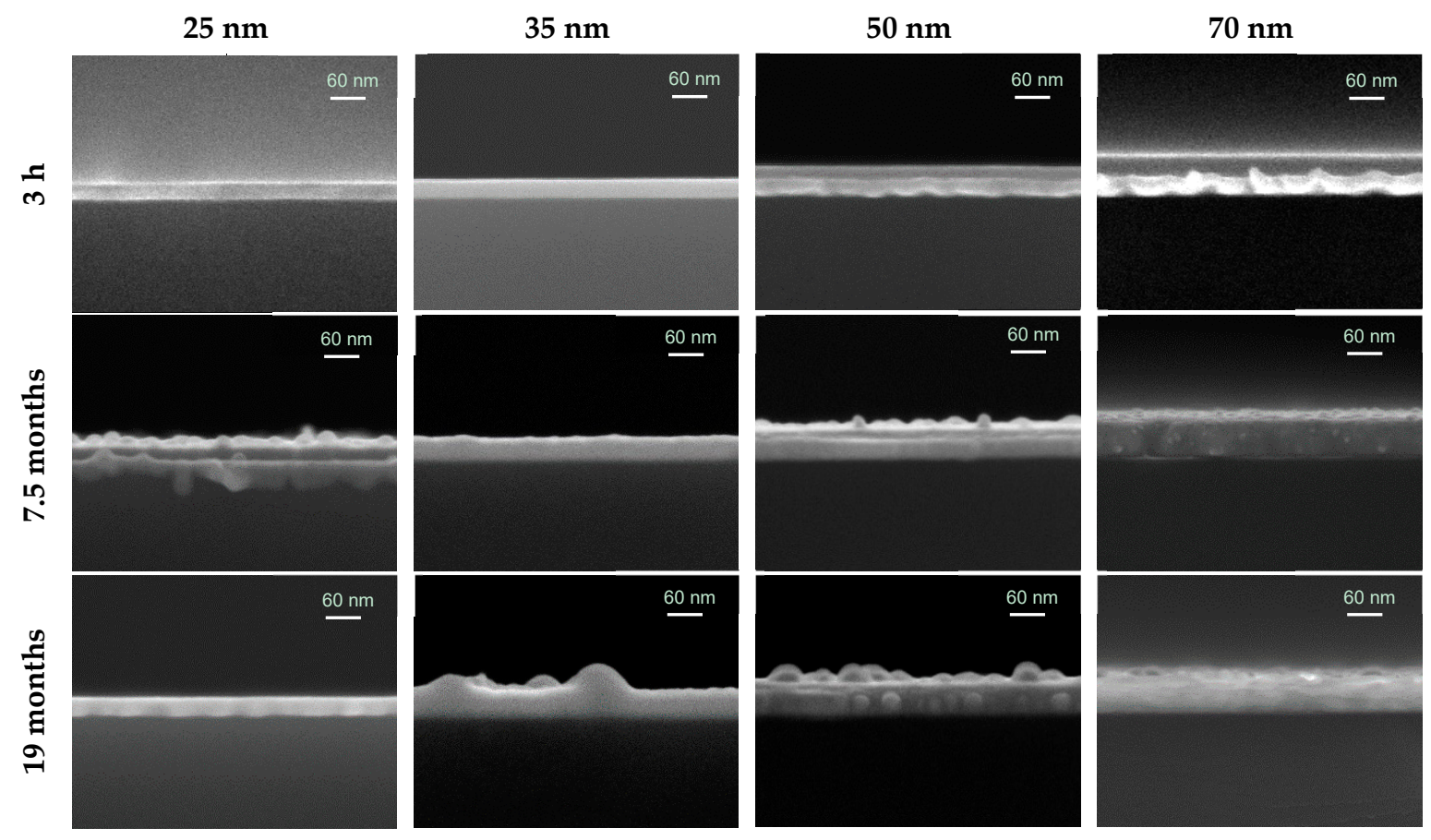

Figure 4. SEM cross section images of silver films of different thickness over time.

Both SEM cross sections (Figure 4) and stylus profiler measurements (Figure 5) shows that silver films surface root-mean-square (RMS) roughness is increased monotonically over 19 months. Looking precisely at the SEM cross sections, one can see that very large (order of half film thickness) defects appear on the films' surface after 19 months. It should be noted that these large defects on the first 
plan of SEM images (35 nm thick silver film after 19 months, Figure 4) could be the result of stress relaxation during substrate cleaving process for cross section measurements. One piece of evidence for this is that these defects could be seen on the cleaved (fresh) sidewall surface of $50 \mathrm{~nm}$ thick film after 19 months, for example.

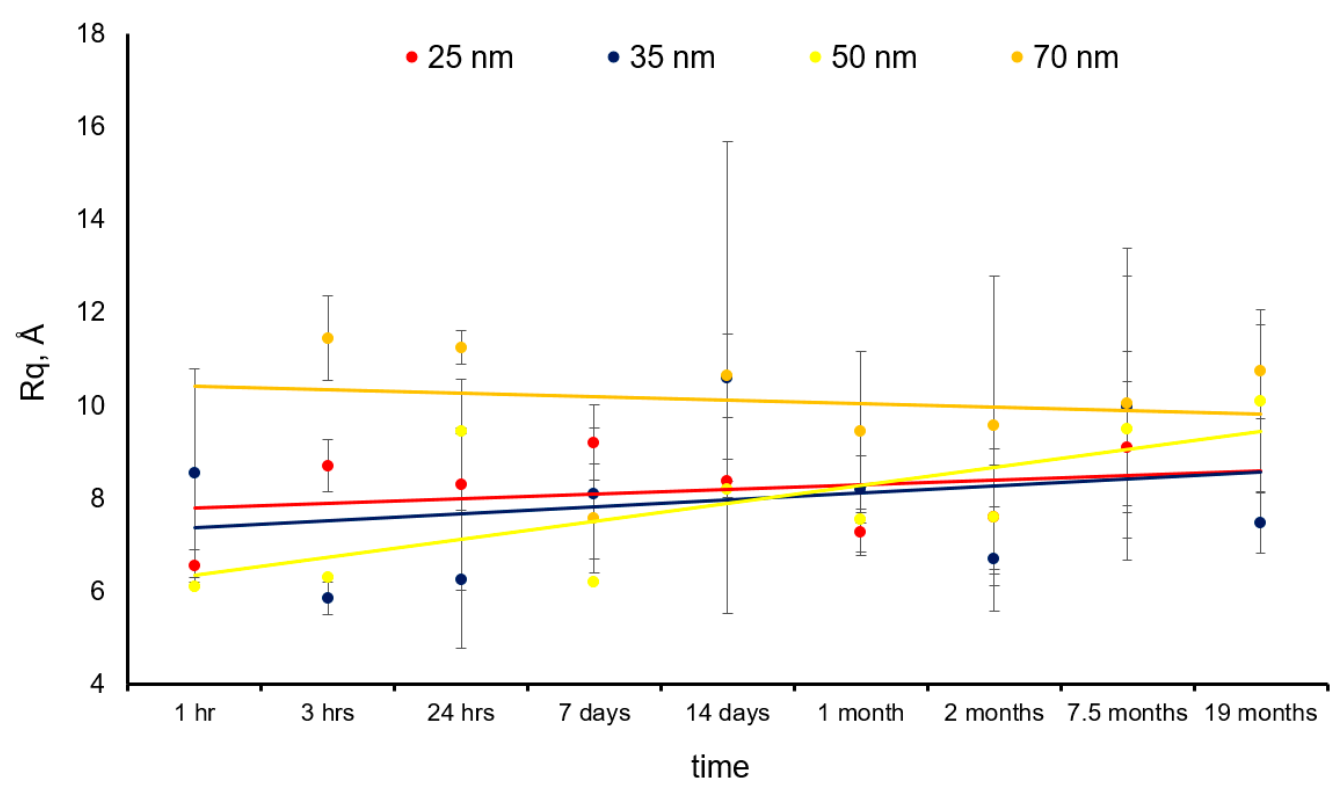

Figure 5. Time evolution of the RMS roughness of Ag thin films of different thicknesses.

The original films were examined by the EBSD method (Figure 6), which confirms that all the films have a single-crystalline structure with small angles of terraces disorientation.

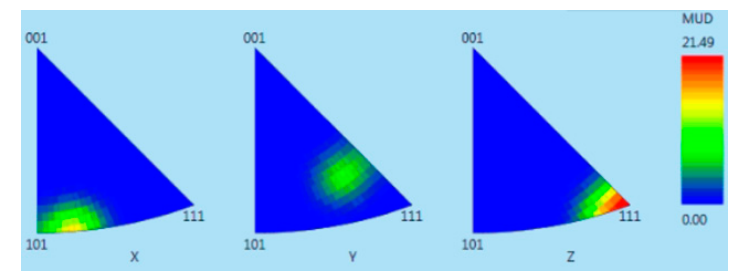

(a) $\operatorname{Ag} 25 \mathrm{~nm}$

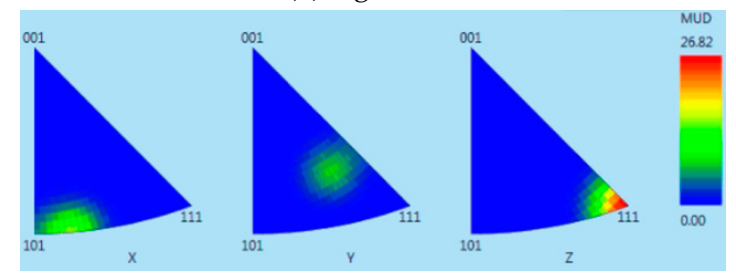

(c) Ag $50 \mathrm{~nm}$

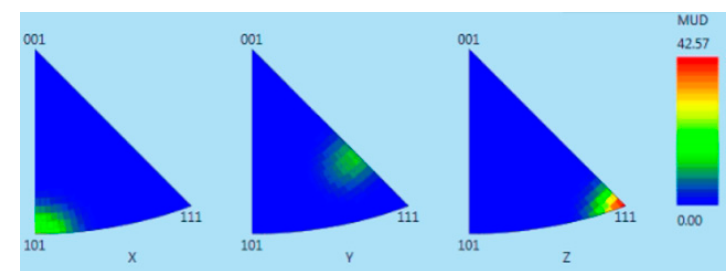

(b) $\operatorname{Ag} 35 \mathrm{~nm}$

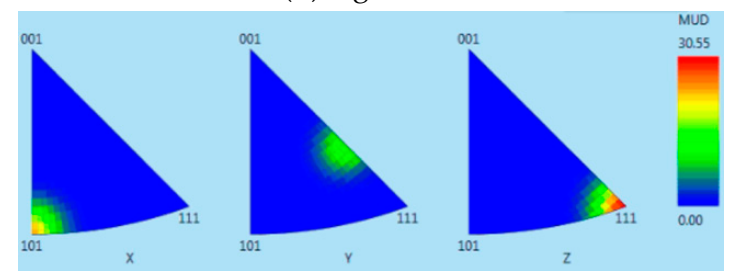

(d) $\operatorname{Ag} 70 \mathrm{~nm}$

Figure 6. Inverse pole figures of $<111>$ silver structure. (a) $25 \mathrm{~nm}$, (b) $35 \mathrm{~nm}$, (c) $50 \mathrm{~nm}$, (d) $70 \mathrm{~nm}$.

\subsection{Optical Constants}

As a result of modeling the ellipsometric data, the dielectric constant dispersions of thin silver films were determined. For simulations, the film array was divided into the main film and the roughness layer (Figure 7) according to SEM cross section data. The main film was simulated using the Brendel-Bormann model [34]. For roughness simulation the Bruggeman model was used [35]. This particular model can be used when inclusions are macroscopic particles, and the matrix cannot be distinguished. Figures 8-11 show two sets of $\varepsilon^{\prime \prime}$ dispersions corresponding to the main layer of the films (a) and the roughness layer (b). 


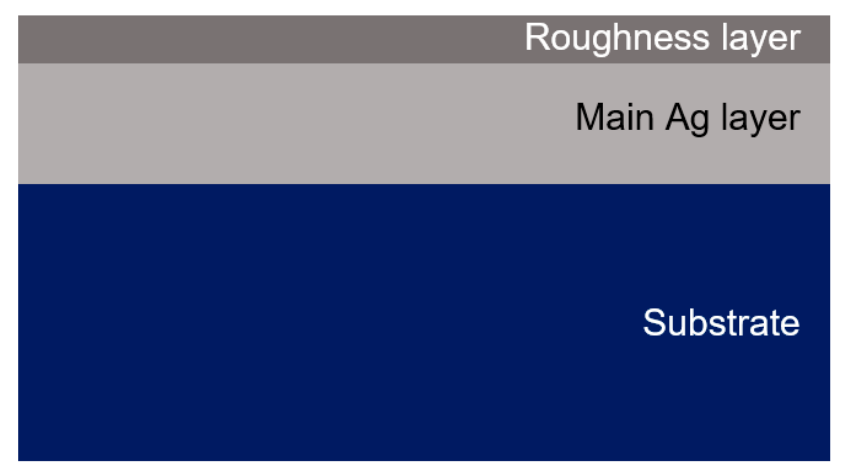

Figure 7. Thin film array at degradation process (model for $\varepsilon^{\prime \prime}$ calculation).
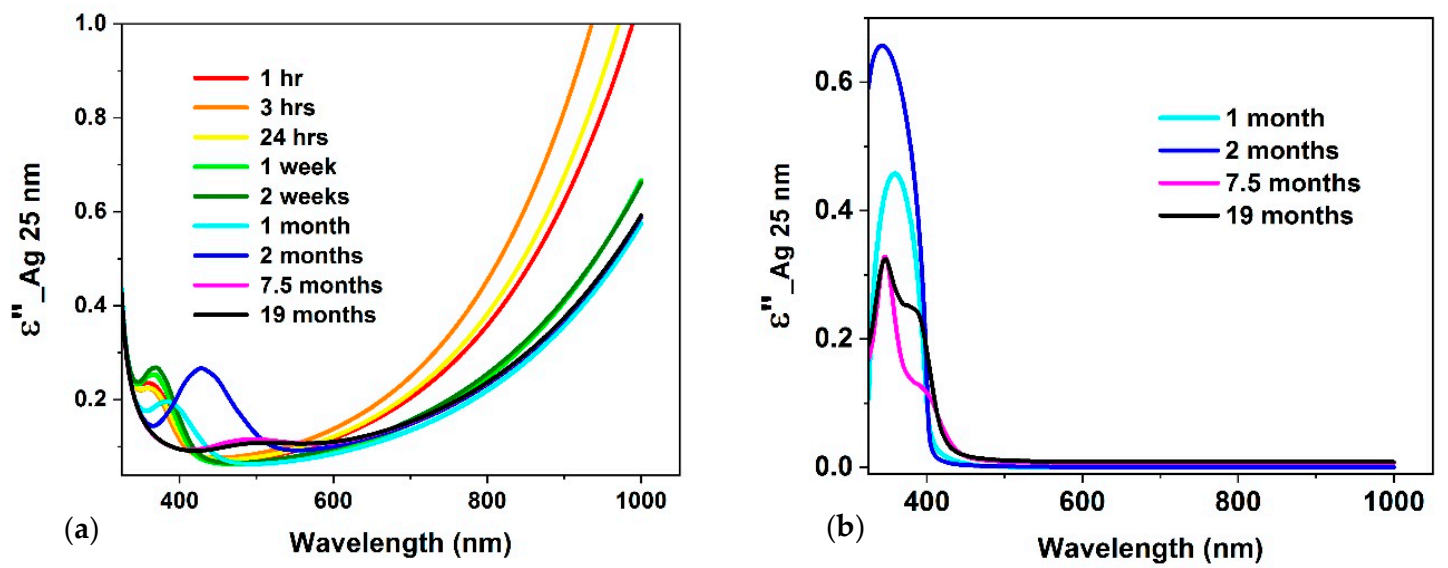

Figure 8. Dielectric permittivity imaginary part evolution for the $25 \mathrm{~nm} \mathrm{Ag}$ thin films: (a) main layer; (b) roughness layer in the range of 330-1000 $\mathrm{nm}$.
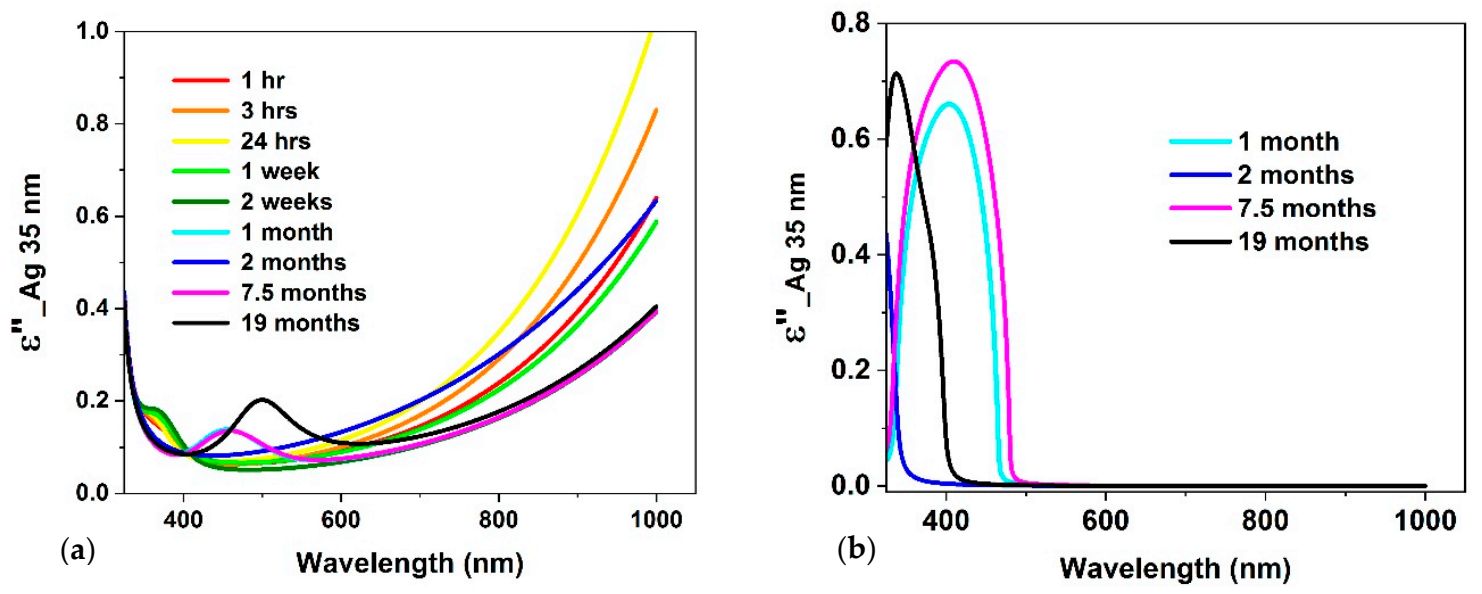

Figure 9. Dielectric permittivity imaginary part evolution for the $35 \mathrm{~nm}$ Ag thin films: (a) main layer; (b) roughness layer in the range of 330-1000 $\mathrm{nm}$. 

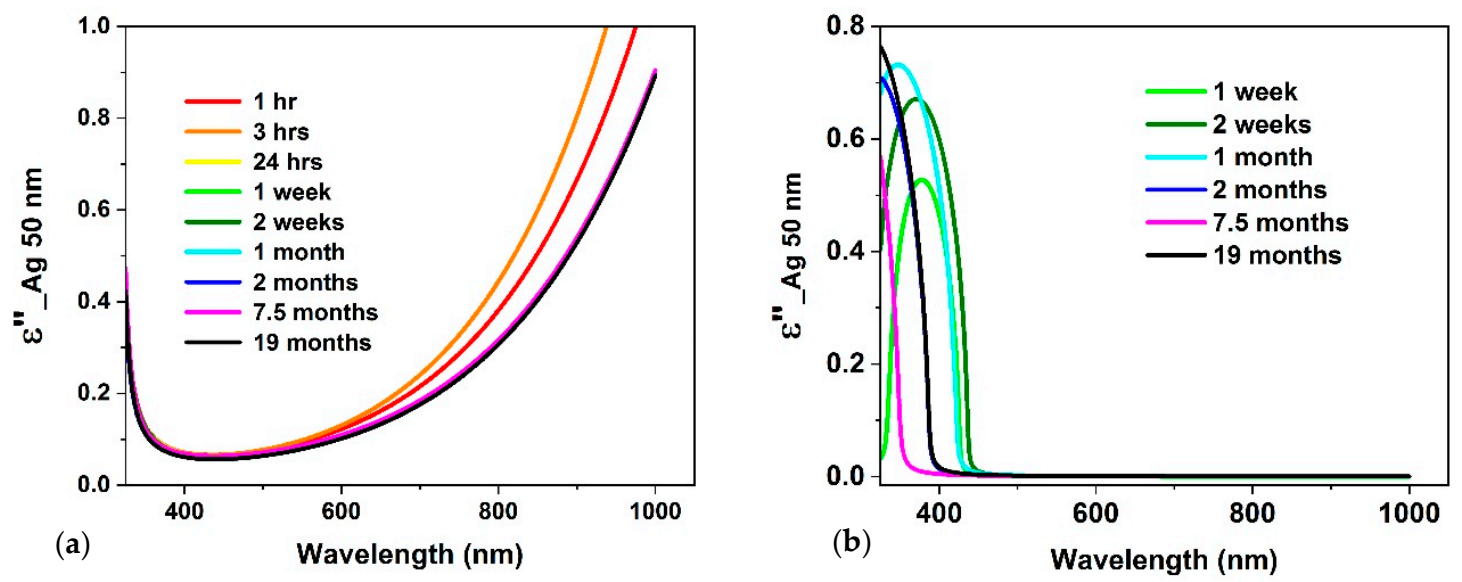

Figure 10. Dielectric permittivity imaginary part evolution for the $50 \mathrm{~nm}$ Ag thin films: (a) main layer; (b) roughness layer in the range of 330-1000 nm.
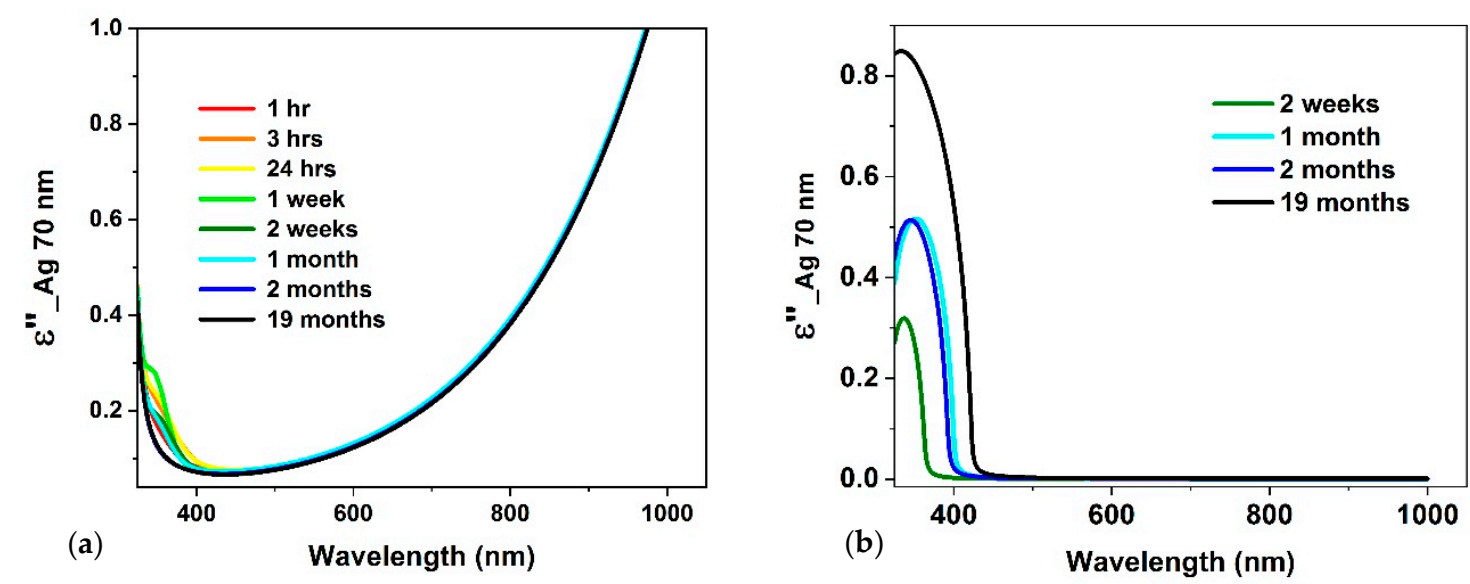

Figure 11. Dielectric permittivity imaginary part evolution for the $70 \mathrm{~nm}$ Ag thin films: (a) main layer; (b) roughness layer in the range of 330-1000 $\mathrm{nm}$.

As shown in Figures 8-11, $\varepsilon^{\prime \prime}$ values (responsible for the losses) at longer wavelengths (600-1000 nm) change over time for all the samples. For the $25 \mathrm{~nm}$ film, the losses increase during the first hours right after the deposition process, but then decrease and practically do not change after 1 month, remaining around 0.6 (hereinafter, at $1000 \mathrm{~nm}$ ) until the expiration of 19 months. For the $35 \mathrm{~nm}$ film, the same trend, except for 2 months, when $\varepsilon^{\prime \prime}$ increased, is observed. As a result, the value $\varepsilon^{\prime \prime}$ decreases down to 0.4 in 7.5 months. For the $50 \mathrm{~nm}$ film, $\varepsilon^{\prime \prime}$ values stabilized even faster. After $24 \mathrm{~h}$, they stopped changing and stabilized at 0.9 . Finally, $\varepsilon^{\prime \prime}$ values of the $70 \mathrm{~nm}$ film exceed 1.0 right after the deposition process and do not change over time. In general, we can see that the thicker the film, the faster $\varepsilon^{\prime \prime}$ values at longer wavelengths stop changing over time. It should be noted that the effect of the roughness layer is negligible in the considered wavelength range, because of the lack of absorption in the roughness layer as seen from the spectrum $\varepsilon^{\prime \prime}(b)$.

Changes at the wavelengths around 350-600 nm have a more complex nature because an interband transition occurs here.

At the same time, the absorption values of the roughness layer are nonzero. For the $25 \mathrm{~nm}$ film, the interband transition shifts towards longer wavelengths up to $500 \mathrm{~nm}$ after 19 months, where the plasmon resonance of the roughness layer is absent. The observed shift can be explained by the growth of size and number of the main film layer defects, which can be seen from the SEM image in Figures 2 and 3 (holes appear). It was observed in [36] that the defects influence the interband transition position (Lorentz peak), leading to the amplitude increase and the interband transition shift. 
However, the plasmon resonance position in the roughness layer indicates that some defects will cause absorption peaking at $350 \mathrm{~nm}$. The shape, amplitude and maximum position of the Lorentz band is intrinsically connected to the RMS-to-thickness ratio and originates not only from the contribution of the interband transitions in the Brillouin zone, but also from the intraband transitions as well as surface plasmon-polariton excitations [32]. For SCULL films the RMS-to-thickness ratio is lower and thus intraband transitions and localized plasmon coupling are limited. This results in a narrow and diminished Lorentz-shaped band.

For the $35 \mathrm{~nm}$ film, we observe a similar tendency, when starting from 1 month, the interband transition position in the main film layer is shifted to the wavelength of $450 \mathrm{~nm}$, which is explained by defects size growth on the surface, forming a rough layer. The peaks of the roughness layer for 1 and 7.5 months are characterized by the maximum at $400 \mathrm{~nm}$. It indicates the presence of various-size defects, which leads to absorption existence in the entire range of $350-550 \mathrm{~nm}$. In the plots for 19 months of observations, we see the emergence of a significant interband transition peak with the increased amplitude right-shifted to $500 \mathrm{~nm}$. However, there was no roughness effect, consequently, it is completely determined by the defects of the main film. The influence of its roughness layer, as in the previous two months, is left-shifted with a peak at $350 \mathrm{~nm}$.

For the $50 \mathrm{~nm}$ film, no interband transition peaks are observed, which means the morphological defects mainly manifest themselves in the dispersion of the roughness layer. Thus, the defects acquire such a shape that can be completely attributed to the roughness layer, which can seen in the SEM cross section images for these films. This phenomenon is especially visible in the image for the $50 \mathrm{~nm}$ film at 19 months. The absorption bands of the roughness layers for the $50 \mathrm{~nm}$ film are characterized by a great variety ranging from $330 \mathrm{~nm}$ to $400 \mathrm{~nm}$.

For the $70 \mathrm{~nm}$ film, an interband transition in the main layer was observed during the first month. After the first month, the transition disappeared. From the SEM cross section images analysis, we conclude that morphological defects form a distinct layer of roughness over time and change optical properties of the roughness layer. At the same time, the absorption bands of the $70 \mathrm{~nm}$ thick film roughness layers lie close to each other in the range of 350-360 nm.

The roughness thickness and silver/air ratio used in calculation have been estimated from the SEM cross section images of the films.

\section{Discussion}

The chemical composition of silver films surface was investigated using Auger electron spectroscopy (Figure S2, Supplementary Materials). However, no bonds of silver with active chemical elements ( $\mathrm{AgCl}, \mathrm{Ag}_{2} \mathrm{~S}, \mathrm{Ag}_{2} \mathrm{O}, \mathrm{Ag}_{2} \mathrm{CO}_{3}$ ) were observed in the films. We assume that the degradation mechanism through the formation of chemical compounds is not dominant when considering the epitaxial silver films under investigation. Based on the fact that there are a lot of cracks in the 25 and $70 \mathrm{~nm}$ films, we can assume that silver reacts with the environment locally in the points where the concentration of defects is the highest (terraces boundaries of single-crystals).

We assume that internal residual stress (i.e., stress arising during the growth of films) is the key degradation factor. During the deposition of the films with a high mobility of adatoms ( $\mathrm{Ag}, \mathrm{Au}, \mathrm{Al}, \mathrm{Cu}$ ) a complex stress behavior occurs, where the maximum of tensile stresses (stress-thickness is in range of $2.0-2.5 \mathrm{~N} / \mathrm{m}$ ) is observed during the coalescence of islands (in the thicknesses range of 10-20 nm). Then, during the solid film formation, compressive stresses in the new layers of silver are increased [37]. As the thickness increases, the total stress of the film moves from tension to compression [37]. When the deposition process is finished, the compressive stresses decrease (structure relaxation occurs). Stress in the surface layers decreases first (fast relaxation-for the $100 \mathrm{~nm}$ films, stress-thickness is rising from $-2 \mathrm{~N} / \mathrm{m}$ to $2 \mathrm{~N} / \mathrm{m}$ ) followed by the stresses in the volume (slow relaxation—stress-thickness is rising by fractions of $\mathrm{N} / \mathrm{m}$ ). In a few days, the film almost completely relaxes, but residual stresses remain. They are concentrated in the areas with the biggest number of defects (dislocations between regions of a single-crystal). Single-crystalline silver films with a thickness of $25 \mathrm{~nm}$ obviously have more 
defects, due to the fact that the thickness of the films lays between the island film and the continuous one. At this thickness, the stresses are not compensated and are maximally tensile [38]. Films ranging from 35 to $50 \mathrm{~nm}$ thick correspond to the stage of partial stress compensation. Starting from certain films thickness, the character of stress in the films changes due to partial compensation and local deformation. In this case, a grid of stress distribution is formed by a scale smaller than the terrace size. It leads to the activation of surface diffusion in relation to diffusion along the boundaries of terraces. In this regard, we observed an increase in the roughness lateral size on the film with its height decrease (Figures 2 and 5). According to Chason et al. [38], the most developed explanation for the emergence of compressive stresses in a growing film is the theory of atoms implantation into the grain boundaries, which was proposed in many works [39-41]. It can be also traced in our experimental results. Electron microscopy shows the emergence of volumetric structures on the surface in the most defective places (Figures 2 and 3), which may consist of atoms trapped from the boundaries.

Ellipsometry results show an overall decrease in the film absorption, but also the appearance of the peaks in the visible range (Figures 8-11). This may indicate a crystalline structure improvement in the bulk, due to defects redistribution and appearance of semi-continuous structures on the surface, which grow over time (in the absorption spectrum, the peaks are shifted and change its shape). Observed surface changes can also be explained by the tendency of flat silver structures to dewetting. Normally, dewetting is observed either at elevated temperatures (even for the films thinner than $35 \mathrm{~nm}$ [29]) or at room temperature in the thin layers (about 10-20 ML) [27]. We consider dewetting as one of the mechanisms of silver degradation combined with the structure relaxation of internal stress. Dewetting occurs in three stages [30]: 1—formation of holes in the thin film; 2-growth of the holes in the mass transfer process; 3-film cracks. In this case, even in a single-crystalline film, the holes appear in the most defective places (at the joints of terraces or at the other point defects).

Starting from a $70 \mathrm{~nm}$ film, the change in the behavior of the surface morphology of the films with time (the appearance of recesses, craters, and cracks-Figure 3) can be explained by processes similar to dewetting, due to a threshold increase in the ration of defective areas to the area of terraces.

\section{Conclusions}

In this work, we systematically investigate the degradation of 25-70 nm thick single-crystalline silver films deposited by electron-beam evaporation over almost two years. Surface morphology, crystalline structure and optical characteristics of our films were measured using ultra high-resolution scanning electron microscopy, stylus profilometry, SEM EBSD technique and spectroscopic ellipsometry under standard laboratory conditions. The initial extremely high surface and crystalline structure quality of single-crystalline silver films is guaranteed by well established SCULL process [33], that provide us to observe the basic reasons of silver degradation (film quality independent). According to our results, the internal film energy accumulated via film stress mechanism is one of the key initiators of degradation in single-crystalline silver films. Together with the strong dewetting naturally specific to silver, it leads to dramatic hole-like formations through film degradation after around one year in the atmosphere. It is worth noticing that with careful thickness choice, the SCULL single-crystalline silver film retains its structural and optical quality over 1-2 years, which is opposite to commonly held opinion on silver film short-term degradation.

After 19 months in the atmosphere, bulk structures (volumetric inhomogeneities) appear on the surface of silver films. We can even observe through-depth defects (holes) in $25 \mathrm{~nm}$ thick film, and the emergence of voids (not full depth holes) in $70 \mathrm{~nm}$ thick films. These structures are associated with the relaxation of internal stresses combined with dewetting. Single-crystalline silver films deposited using the SCULL technology of 35-50 nm thickness demonstrated the best stability in terms of structural and optical properties degradation. We have shown that the number of structural defects (grain boundaries and joints of terraces) is among the key factors that influence the degradation intensity of silver films. Ellipsometry results revealed a decrease in films' optical losses with time in the wavelength range $600-1000 \mathrm{~nm}$, as well as the emergence of peaks in the range $350-600 \mathrm{~nm}$, and the increase in the 
roughness layer associated with the emergence and growth of the bulk structures on the surface. In this case, the 25 and $70 \mathrm{~nm}$ films are characterized by increasing losses in the formed roughness layer with time.

We proposed the mechanism explaining changes in the thin metal films characteristics. The changes are associated with the distribution of internal stresses as well as silver layers tendency to dewetting. As part of the proposed mechanism, the nature of roughness dependencies on the exposure time for different film thicknesses and the improvement of their optical characteristics was explained. Interestingly, some SCULL single-crystalline films improve their crystalline structure over time with a simultaneous change in the surface morphology. This is the example of benefit stress relaxation in epitaxial silver films, and this process is completely reproducible. The SCULL single-crystalline silver films of 35-50 nm thickness demonstrated the highest stability over two years.

Supplementary Materials: The following are available online at http://www.mdpi.com/2079-6412/10/10/911/s1, Figure S1: SEM images of Ag thin films, Figure S2: AES of Ag thin film.

Author Contributions: Conceptualization, I.A.R. (Ilya A. Rodionov) and A.S.B.; methodology, A.S.B., D.O.M. and T.G.K.; analyze and validation of the optical properties, I.A.B.; analyze and validation of the surface structure properties, I.A.R. (Ilya A. Ryzhikov) and E.S.L.; over-time investigation, A.I.I., O.S.S., E.V.S., K.A.B. and Z.I.; resources, E.S.L., A.S.B. and I.A.R. (Ilya A. Rodionov); data curation, E.S.L. and A.I.I.; writing-original draft preparation, E.S.L., I.A.R. (Ilya A. Ryzhikov) and A.S.B.; writing-review and editing, A.I.I. and I.A.R. (Ilya A. Rodionov); visualization, E.S.L. and O.S.S.; supervision, I.A.R. (Ilya A. Rodionov) and I.A.R. (Ilya A. Ryzhikov); project administration, I.A.R. (Ilya A. Rodionov) All authors have read and agreed to the published version of the manuscript.

Funding: This research received no external funding.

Acknowledgments: Samples were made and measured at the BMSTU Nanofabrication Facility (FMN Laboratory, FMNS REC, ID 74300).

Conflicts of Interest: The authors declare no conflict of interest.

\section{References}

1. Baburin, A.S.; Merzlikin, A.M.; Baryshev, A.V.; Ryzhikov, I.A.; Panfilov, Y.V.; Rodionov, I.A. Silver-based plasmonics: Golden material platform and application challenges. Opt. Mater. Express 2019, 9, 611-642. [CrossRef]

2. West, P.R.; Ishii, S.; Naik, G.V.; Emani, N.K.; Shalaev, V.M.; Boltasseva, A. Searching for better plasmonic materials. Laser Photonics Rev. 2010, 4, 795-808. [CrossRef]

3. Yankovskii, G.M.; Komarov, A.V.; Puz'ko, R.S.; Baryshev, A.V.; Afanas'ev, K.N.; Boginskaya, I.A.; Bykov, I.V.; Merzlikin, A.M.; Rodionov, I.A.; Ryzhikov, I.A. Structural and optical properties of single and bilayer silver and gold films. Phys. Solid State 2016, 58, 2503-2510. [CrossRef]

4. Haffner, C.; Chelladurai, D.; Fedoryshyn, Y.; Josten, A.; Baeuerle, B.; Heni, W.; Watanabe, T.; Cui, T.; Cheng, B.; Saha, S.; et al. Low-loss plasmon-assisted electro-optic modulator. Nature 2018, 556, 483-486. [CrossRef]

5. Ma, R.M.; Yin, X.B.; Oulton, R.F.; Sorger, V.J.; Zhang, X. Multiplexed and Electrically Modulated Plasmon Laser Circuit. Nano Lett. 2012, 12, 5396-5402. [CrossRef] [PubMed]

6. Danqing, W.; Weijia, W.; Knudson, M.P.; Schatz, G.C.; Odom, T.W. Structural Engineering in Plasmon Nanolasers. Chem. Rev. 2018, 118, 2865-2881.

7. Nahata, A.; Linke, R.A.; Ishi, T.; Ohashi, K. Enhanced nonlinear optical conversion from a periodically nanostructured metal film. Opt. Lett. 2003, 28, 423-425. [CrossRef] [PubMed]

8. Eisenbraum, E.T.; Klaver, A.; Patel, Z.; Nuesca, G.; Kaloyeros, A.E. Low temperature metalorganic chemical vapor deposition of conformal silver coatings for applications in high aspect ratio structures. J. Vac. Sci. Technol. B 2001, 19, 585. [CrossRef]

9. Baburin, A.S.; Kalmykov, A.S.; Kirtaev, R.V.; Negrov, D.V.; Moskalev, D.O.; Ryzhikov, I.A.; Melentiev, P.N.; Rodionov, I.A.; Balykin, V.I. Toward a theoretically limited SPP propagation length above two hundred microns on an ultra-smooth silver surface. Opt. Mater. Express 2018, 8, 3254-3261. [CrossRef] 
10. Rodionov, I.A.; Baburin, A.S.; Rizhikov, I.A.; Trofimov, I.V.; Philippov, I.A.; Gabidulin, A.R.; Dobronosova, A.A.; Vinogradov, A.P.; Zverev, A.V.; Ivanov, A.I.; et al. Mass production compatible fabrication techniques of single-crystalline silver metamaterials and plasmonics devices. In Proceedings of the Metamaterials, Metadevices, and Metasystems 2017, San Diego, CA, USA, 6-10 August 2017.

11. Melentiev, P.; Kalmykov, A.; Gritchenko, A.; Afanasiev, A.; Balykin, V.; Baburin, A.S.; Ryzhova, E.; Filippov, I.; Rodionov, I.A.; Nechepurenko, I.A.; et al. Plasmonic nanolaser for intracavity spectroscopy and sensorics. Appl. Phys. Lett. 2017, 111, 213104. [CrossRef]

12. Baburin, A.S.; Ivanov, A.I.; Trofimov, I.V.; Dobronosova, A.A.; Melentiev, P.N.; Balykin, V.I.; Moskalev, D.O.; Pishchimova, A.A.; Ganieva, L.A.; Ryzhikov, I.A.; et al. Highly directional plasmonic nanolaser based on high-performance noble metal film photonic crystal. In Proceedings of the Nanophotonics VII. International Society for Optics and Photonics, Strasbourg, France, 22-26 April 2018; p. 10672.

13. Homola, J. Surface plasmon resonance sensors for detection of chemical and biological species. Chem. Rev. 2008, 108, 462-493. [CrossRef] [PubMed]

14. Baburin, A.S.; Gritchenko, A.S.; Orlikovsky, N.A.; Dobronosova, A.A.; Rodionov, I.A.; Balykin, V.I.; Melentiev, P.N. State-of-the-art plasmonic crystals for molecules fluorescence detection. Opt. Mater. Express 2019, 9, 1173-1179. [CrossRef]

15. Lagarkov, A.; Boginskaya, I.; Bykov, I.; Budashov, I.; Ivanov, A.; Kurochkin, I.; Ryzhikov, I.; Rodionov, I.; Sedova, M.; Zverev, A.; et al. Light localization and SERS in tip-shaped silicon metasurface. Opt. Express 2017, 25, 17021-17038. [CrossRef] [PubMed]

16. Gong, H.M.; Zhou, L.; Su, X.R.; Xiao, S.; Liu, S.D.; Wang, Q.Q. Illuminating Dark Plasmons of Silver Nanoantenna Rings to Enhance Exciton-Plasmon Interactions. Adv. Funct. Mater. 2009, 19, 298-303. [CrossRef]

17. Bogdanov, S.I.; Shalaginov, M.Y.; Lagutchev, A.S.; Chiang, C.C.; Shah, D.; Baburin, A.S.; Ryzhikov, I.A.; Rodionov, I.A.; Kildishev, A.V.; Boltasseva, A.; et al. Ultrabright room-temperature sub-nanosecond emission from single nitrogen-vacancy centers coupled to nanopatch antennas. Nano Lett. 2018, 18, 4837-4844. [CrossRef]

18. High, A.A.; Devlin, R.C.; Dibos, A.; Polking, M.; Wild, D.S.; Perczel, J.; de Leon, N.P.; Lukin, M.D.; Park, H. Visible-frequency hyperbolic metasurface. Nature 2015, 522, 192-196. [CrossRef]

19. Nepal, D.; Park, K.; Vaia, R.A. High-Yield Assembly of Soluble and Stable Gold Nanorod Pairs for High-Temperature Plasmonics. Small 2012, 8, 1013-1020. [CrossRef]

20. Park, J.H.; Ambwani, P.; Manno, M.; Lindquist, N.C.; Nagpal, P.; Oh, S.H.; Leighton, C.; Norris, D.J. Single-crystalline silver films for plasmonics. Adv. Mater. 2010, 24, 3988-3992. [CrossRef]

21. Breslin, C.B.; Macdonald, D.D. The influence of Uv light on the dissolution and passive behavior of copper-containing alloys in chloride solution. Electrochim. Acta 1998, 44, 643-651. [CrossRef]

22. Burleigh, T.D.; Ruhe, C.; Forsyth, J. Photo-corrosion of different metals during long-term exposure to ultraviolet light. Corros. Sci. 2003, 59, 774-779. [CrossRef]

23. Keast, V.J.; Myles, T.A.; Shahcheraghi, N.; Cortie, M.B. Corrosion processes of triangular silver nanoparticles compared to bulk silver. J. Nanopart. Res. 2016, 18, 45. [CrossRef]

24. Oates, T.W.H.; Losurdo, M.; Noda, S.; Hinrichs, K. The effect of atmospheric tarnishing on the optical and structural properties of silver nanoparticles. J. Phys. D Appl. Phys. 2013, 46, 145308. [CrossRef]

25. Águas, H.; Silva, R.J.C.; Viegas, M.; Pereira, L.; Fortunato, E.; Martins, R. Study of environmental degradation of silver surface. Phys. Status Solidi C 2008, 5, 1215-1218. [CrossRef]

26. Trautmann, S.; Dathe, A.; Csáki, A.; Thiele, M.; Müller, R.; Fritzsche, W.; Stranik, O. Time-Resolved Study of Site-Specific Corrosion in a Single Crystalline Silver Nanoparticle. Nanoscale Res. Lett. 2019, $14,240$. [CrossRef] [PubMed]

27. Sanders, C.E.; Zhang, C.; Kellogg, G.L.; Shih, C.K. Role of thermal processes in dewetting of epitaxial Ag(111) film on Si(111). Surf. Sci. 2014, 630, 168-173. [CrossRef]

28. Wang, X.; Santschi, C.; Martin, O.J.F. Strong Improvement of Long-Term Chemical and Thermal Stability of Plasmonic Silver Nanoantennas and Films. Small 2017, 13, 1700044. [CrossRef] [PubMed]

29. Kim, H.C.; Alford, T.L.; Allee, D.R. Thickness Dependence on the Thermal Stability of Silver Thin Films. Appl. Phys. Lett. 2002, 81, 4287-4289. [CrossRef]

30. Thompson, C.V. Solid-State Dewetting of Thin Films. Annu. Rev. Mater. Res. 2012, 42, 399-434. [CrossRef] 
31. Chi, L.; Bassim, N. Ag Thin Film Dewetting Prevention by Ion Implantation. Adv. Mater. Interfaces 2019, 6, 1900108. [CrossRef]

32. Ciesielski, A.; Skowronski, L.; Trzcinski, M.; Szoplik, T. Controlling the optical parameters of self-assembled silver films with wetting layers and annealing. Appl. Surf. Sci. 2017, 421, 349-356. [CrossRef]

33. Rodionov, I.A.; Baburin, A.S.; Gabidullin, A.R.; Maklakov, S.S.; Peters, S.; Ryzhikov, I.A.; Andriyash, A.V. Quantum Engineering of Atomically Smooth Single Crystalline Silver Films. Sci. Rep. 2019, 9, 12232. [CrossRef] [PubMed]

34. Brendel, R.; Bormann, D. An infrared dielectric function model for amorphous solids. J. Appl. Phys. 1992, 71, 1-6. [CrossRef]

35. Bruggeman, D.A.G. The calculation of various physical constants of heterogeneous substances. I. The dielectric constants and conductivities of mixtures composed of isotropic substances. Ann. Phys. 1935, 23, 636-664. [CrossRef]

36. Li, X.D.; Chen, T.P.; Liu, Y.; Leong, K.C. Influence of localized surface plasmon resonance and free electrons on the optical properties of ultrathin Au films: A study of the aggregation effect. Opt. Express 2014, 22, 5124-5132. [CrossRef] [PubMed]

37. Abermann, R. Measurements of the intrinsic stress in thin metal films. Vacuum 1990, 41, 1279-1282. [CrossRef]

38. Chason, E.; Guduru, P.R. Tutorial: Understanding residual stress in polycrystalline thin films through real-time measurements and physical models. J. Appl. Phys. 2016, 119, 191101. [CrossRef]

39. Leib, J.; Mo“nig, R.; Thompson, V. Direct Evidence for Effects of Grain Structure on Reversible Compressive Deposition Stresses in Polycrystalline Gold Films. Phys. Rev. Lett. 2009, 102, 256101. [CrossRef]

40. Nix, W.D.; Clemens, B.M. Crystallite coalescence: A mechanism for intrinsic tensile stresses in thin films. J. Mater. Res. 1999, 14, 3467-3473. [CrossRef]

41. Spaepen, F. Interfaces and stresses in thin films. Acta Mater. 2000, 48, 31-42. [CrossRef]

(C) 2020 by the authors. Licensee MDPI, Basel, Switzerland. This article is an open access article distributed under the terms and conditions of the Creative Commons Attribution (CC BY) license (http://creativecommons.org/licenses/by/4.0/). 